\title{
A LOW-RESOLUTION SPECTROPHOTOMETER FOR MEASURING THE INTEGRATED COLOUR OF GALAXIES*
}

\author{
K. D. RAKOS, W. W. WEISS, S. MÜLLER, R. PRESSBERGER, \\ and \\ P. WACHTLER \\ Institute for Astronomy, University of Wien, Austria \\ J. M. SCHOMBERT \\ Dept. Astronomy, University of Michigan, Ann Arbor, Michigan, U.S.A. \\ and \\ T. J. KREIDL \\ Lowell Observatory, Flagstaff, Arizona, U.S.A.
}

\begin{abstract}
The spectrophotometer presented here combines the advantage of aperture photometry with spectrophotometry to obtain colours of extended objects over the range of 3500-6000 $\AA$. The spectrophotometric resolution matches the resolution of intermediate-band interference filters of about $140 \AA$ FWHM.
\end{abstract}

\section{General Description}

The top end of the instrument consists of a set of apertures (up to $12 \mathrm{~mm}$ diameter). One fixed aperture is for the object, the second of the same size is located on a movable arm for sky measurements. In the first mode, a $f / 2$ fused silica biconvex lens located behind each aperture, images the entrance pupil of the telescope onto fiber cables. In the second mode, a fused silica planoconvex lens images the entrance pupil of the telescope onto a small $f / 2$ fused silica biconvex lens. This second lens re-images the focus plane of the telecope onto fiber bundles. The second mode of operation provides a $f / 2$ cone of light on the grating independent of the angular size of the object. This may be important in the case that the blaze efficiency of the grating is not uniform or in the case of spectrophotometry in crowded fields.

The circular geometry for the telescope end of the fiber bundle is modified to a rectangular geometry for the other end, thus providing a slit for the subsequent spectrograph. The fiber bundle consists of 55 single-fused silica optical fibers. The numerical aperture is 0.37 and the core to clading ratio is 0.200 to $0.230 \mathrm{~mm}$.

An aberration-corrected holographic concave grating is used to build the spectrometer. The grating used in our instrument has 225 groves $\mathrm{mm}^{-1}$ and the focal ratio of $f / 2$. The blank dimension is $108 \mathrm{~mm}$ in diameter. The linear dispersion is $200 \AA \mathrm{mm}^{-1}$. In the design of our instrument, the spectral resolution is governed primarily by the slit width. The effective slit width is $0.7 \mathrm{~mm}$, producing a nominal spectral resolution of

* Paper presented at the 11th European Regional Astronomical Meetings of the IAU on New Windows to the Universe', held 3-8 July, 1989, Tenerife, Canary Islands, Spain. 
$140 \AA$. The resolution is comparable to the FWHM of the intermediate-band filters used in photometric systems. The residual effects introduced by aberration and the curvature of the image plane are small in comparison to the slit width.

The spectrum produced by the grating is recorded by a CCD camera. The spectrum is oversampled, considering the prescribed spectral resolution and the CCD pixel size. The oversampling can be minimized using an additional lens in front of CCD or via pixel binning on the chip. The advantage of our design is that it is ideal for large, low-contrast objects (i.e., low surface brightness galaxies) since a maximum amount of light is imaged onto the slit, along with simultaneous sky measurements with each exposure. The complete description and drawings of the spectrophotometer will be published elsewhere.

\section{Acknowledgement}

We gratefully acknowledge the financial support from the Austrian 'Fonds für Förderung der Wissenschaftlichen Forschung' 